\title{
EchoGéo
}

$56 \mid 2021$

Les dynamiques spatiales contemporaines de la Russie

\section{Pour ou contre la Rénovation? Inégalités résidentielles, mobilisations et appropriations des immeubles khrouchtchéviens à Moscou}

\section{Guenola Inizan}

\section{(2) OpenEdition}

Journals

Édition électronique

URL : https://journals.openedition.org/echogeo/21740

DOI : $10.4000 /$ echogeo.21740

ISSN : 1963-1197

Éditeur

Pôle de recherche pour l'organisation et la diffusion de l'information géographique (CNRS UMR 8586)

Référence électronique

Guenola Inizan, « Pour ou contre la Rénovation? Inégalités résidentielles, mobilisations et appropriations des immeubles khrouchtchéviens à Moscou », EchoGéo [En ligne], 56 | 2021, mis en ligne le 25 juin 2021, consulté le 03 août 2021. URL : http://journals.openedition.org/echogeo/21740 ; DOl : https://doi.org/10.4000/echogeo.21740

Ce document a été généré automatiquement le 3 août 2021

EchoGéo est mis à disposition selon les termes de la licence Creative Commons Attribution - Pas d'Utilisation Commerciale - Pas de Modification 4.0 International (CC BY-NC-ND) 


\title{
Pour ou contre la Rénovation? Inégalités résidentielles, mobilisations et appropriations des immeubles khrouchtchéviens à Moscou
}

\author{
Guenola Inizan
}

L'auteure remercie le Centre d'Études Franco-Russe (CEFR - UMR MEAE CNRS USR 3060) et la Chaire Habiter Ensemble la Ville de Demain du Labex IMU (ANR-10-LABX-0088) pour leur soutien financier, ainsi que la Vysokovsky Graduate School of Urbanism (HSE University) et les habitant.e.s de Bogordskoe pour leur accueil moscovite.

1 Le passage à l'économie de marché a radicalement transformé le centre de Moscou, rapidement devenu support privilégié des activités commerciales et financières de la capitale russe (Kolossov et O'Loughlin, 2004 ; Makhrova et Molodikova, 2006) et terrain de gentrification (Badyina et Golubchikov, 2005 ; Makhrova et Tatarintseva, 2006). Les périphéries de la ville ont également connu de brutales mutations, causées l'implantation rapide de résidences de luxe (Zotova, 2012) ou le développement de vastes projets de promotion immobilière. Parallèlement, dans l'ensemble du tissu urbain, certains aménagements ont révélé une "transition" (entendue dans son acceptation large de production d'une ville « post-soviétique ») parfois conflictuelle et contestée (Argenbright, 2016 ; Fröhlich, 2019). En creux de ces dynamiques spatiales tangibles, les quartiers résidentiels khrouchtchéviens, situés dans l'ensemble de la ville mais concentrés dans un anneau péricentral, constituent des terrains plus discrets de la transition. Pourtant, ces lieux « ordinaires $»^{1}$ émaillant le paysage de la capitale ont aussi absorbé et modelé les transformations sociales post-soviétiques (Amestoy, 2004 ; Reid, 2018). Ils ont notamment été concernés par les transformations de la propriété et de la copropriété, dont les structures légales ont été inégalement appropriées selon les immeubles et les habitants (Chaigneau, 2012 ; Richard, 2014). 
2 Depuis l'annonce du programme Rénovation en février 2017, les quartiers ${ }^{2}$ khrouchtchéviens font face à une dimension plus spectaculaire de la transition postsoviétique. Ce programme vise $5177^{3}$ de ces immeubles et prévoit la construction de logements neufs pour reloger les habitants. Justifié officiellement par l'obsolescence imminente des immeubles, la nécessité de modernisation et de densification de la ville, il s'attaque à une strate urbaine emblématique et souvent présentée comme homogène. En réalité, ces immeubles sont divers, tant par leur architecture (préfabriqués de béton ou immeubles de brique) que par les situations résidentielles qu'ils abritent. La Rénovation suscite ainsi des réactions très différenciées : certains habitants attendent plein d'espoirs sa réalisation, d'autres s'y opposent fermement. Comment expliquer ces réceptions différenciées de la démolition annoncée ? Comment sa contestation prendelle forme localement?

3 Cet article cherche à répondre à ces questions en considérant la Rénovation comme un « événement » (Coing, 1966) révélant, produisant et transformant des appropriations diverses et inégales de l'espace habité. Les habitants réagissent de façon différenciée à la démolition latente, selon ce qu'ils pensent avoir à gagner ou à perdre face au changement. Les pratiques (aménagement, travaux, investissement dans la vie de l'immeuble ou du quartier...) et représentations (attachement, désaffection, familiarité ...) de l'espace habité sont brutalement fragilisées, intensifiées ou altérées. En outre, l'annonce du programme génère des soutiens ou contestations qui s'expriment dans le cadre de l'architecture institutionnelle de la Rénovation (vote, participation à des audiences publiques) sans s'y cantonner. Les rassemblements de milliers de Moscovites dans le centre-ville au printemps 2017 sont les exemples les plus visibles de résistance au programme, mais elles se déclinent sous d'autres modalités (réseaux sociaux, réunions locales...).

4 En exposant les transformations des appropriations habitantes des espaces résidentiels khrouchtchéviens et les formes de mobilisation (individuelles et collectives), dans le contexte de la Rénovation, je cherche, dans la lignée de précédents travaux (Chaigneau, 2012 ; Carton de Grammont, 2013 ; Richard 2014) à analyser la transition urbaine à Moscou non comme un changement linéaire et inéluctable (Rolland, Volin, Coudroy de Lille et Rivière-Honegger, 2017), mais comme un ensemble de relations sociales, de pratiques et de représentations variées et inégales, ici envisagées dans leurs dimensions spatiales. L'article s'appuie sur une enquête de terrain localisée dans un district de l'est moscovite, Bogorodskoe, particulièrement touché par la Rénovation. Elle y a suscité des réactions polarisées et une mobilisation importante des habitants opposés au programme. Pour en rendre compte, je mobilise une série d'entretiens et d'observations ${ }^{4}$, mis en regard avec les décrets définissant le cadre légal du programme, les listes des immeubles concernés et une veille sur les réseaux sociaux et la presse locale.

5 La première partie de l'article revient sur la mise à l'agenda de la démolition des immeubles khrouchtchéviens à Moscou et défend une approche de la transition urbaine par les appropriations et mobilisations habitantes. La deuxième partie expose les modalités du vote "pour» ou «contre» la Rénovation et met en perspective les résultats avec l'inégalité des situations résidentielles et des réactions au programme. Dans une troisième partie, je montre comment la Rénovation a fragilisé les appropriations de l'espace habité, mais a aussi conduit à le réinvestir, en particulier à 
travers la mobilisation des opposants, qui s'est néanmoins heurtée à la diversité des situations résidentielles, traduites en intérêts divergents.

\section{Les quartiers khrouchtchéviens face à la Rénovation: une strate urbaine soviétique habitée en proie à la démolition}

\section{Des terrains discrets de la transition post-soviétique}

6 La transformation de Moscou, ex-capitale soviétique, en "ville capitaliste géante " (Kolossov et O'Loughlin, 2004) a été manifeste dans les espaces centraux et aux seuils de la ville. Le développement d'espaces voués aux activités commerciales (Makhrova et Molodikova, 2006) a augmenté le coût du foncier du centre-ville, qui est aussi devenu de plus en plus sélectif socialement. Concerné par l'éviction de populations pauvres dès les années 1980 (Trouchtchenko, 1992), le centre de Moscou est rapidement devenu le terrain d'une gentrification brutale, fruit d'une alliance entre pouvoirs publics et promoteurs (Golubchikov, Badynia, 2005 ; Makhrova et Tatarintseva, 2006), processus qui a participé à creuser les écarts entre centre et périphérie (Vendina, 2014 ; Marchand, 2010). Néanmoins, du fait de l'héritage de la structure résidentielle soviétique ${ }^{5}$, une certaine cohabitation de populations aux niveaux de vie inégaux y perdure, générant des formes de ségrégation visibles à des échelles fines, comme celle des immeubles (Golubchikov et Makhrova, 2012). Aux seuils de la ville, d'autres signes de l'urbanisation capitaliste sont rapidement apparus. Les résidences fermées de luxe, florissant notamment à l'Ouest de la ville (Zotova, 2012 ; Marchand, 2010) marquent l'émergence d'une classe aisée alors que les nouvelles hautes tours d'habitation sont parfois présentées comme des "ghettos" repoussoirs (Zhelnina, 2019a). Enfin, le développement du « Nouveau Moscou » (illustration 1), annexe territoriale intégrée à la ville en 2012, reflète des motivations politiques et financières des décideurs, dont la cohérence territoriale pose d'autant plus question que le projet ne remporte pas le succès espéré (Argenbright, 2018).

7 Entre les fronts d'urbanisation et le centre-ville, les quartiers khrouchtchéviens (illustration 2), jusqu'ici affectés ponctuellement par les projets d'aménagement, incarnent une dimension plus discrète des transformations post-soviétiques. Ces quartiers ont été construits entre la fin des années 1950 et les années 1960, dans le cadre du programme de construction de logements lancé en 1957 par Nikita Khrouchtchev. Le développement de techniques de préfabrication, avait rendu possible la construction d'immeubles entiers en quelques jours. La standardisation des bâtiments (Solopova, 2021), ainsi que l'implication de citoyens dans la construction (Harris, 2013) avaient permis de produire ces logements à grande échelle. Ainsi des millions de soviétiques ont emménagé dans des nouveaux appartements entre la fin des années 1950 et le début des années 1970 dans toute l'URSS. Ces nouveaux logements symbolisaient l'accès au confort moderne, même si la réalité du sous-équipement des appartements et des quartiers (microraïony) ternissait ce tableau.

8 À Moscou, les quartiers khrouchtchéviens constituaient un front d'urbanisation au moment de leur construction. Ils forment aujourd'hui une couronne péricentrale intégrée dans la ville (illustration 1 ; Gunko et al., 2018). Leur certaine homogénéité paysagère (immeubles de trois ou quatre étages, aux façades simples et parfois 
dégradées, entourés de vastes cours) a été une première fois ébréchée par les tours d'habitation brejnéviennes bâties dans les années 1970 et 1980. Puis elle a été de nouveau troublée dans les années 1990 et 2000 où tout espace non bâti, si exigu qu'il fût, était susceptible d'être perforé par un kiosque, un garage, une haute d'habitation ou toute autre "construction ponctuelle " (totchechtnaya zastroika). Suite à la loi sur la privatisation gratuite des logements occupés de 1992, les appartements de ces immeubles ont été majoritairement privatisés, au compte-gouttes, mais certains demeurent à "baux municipaux ", c'est-à-dire loués à la municipalité6. En outre, le Code du logement de 2005 a encouragé le développement de structures de copropriété à l'échelle des immeubles, les "sociétés de propriétaires de logement", qui ont été inégalement investies par les habitants (Richard, 2013). Les immeubles khrouchtchéviens ont ainsi en partie survécu à la transition politique et économique de Moscou mais ils ont absorbé les transformations structurelles du secteur du logement, en particulier la privatisation et la mise en place du marché immobilier.

Illustration 1 - Démolitions prévues dans le cadre de la Rénovation (2019)

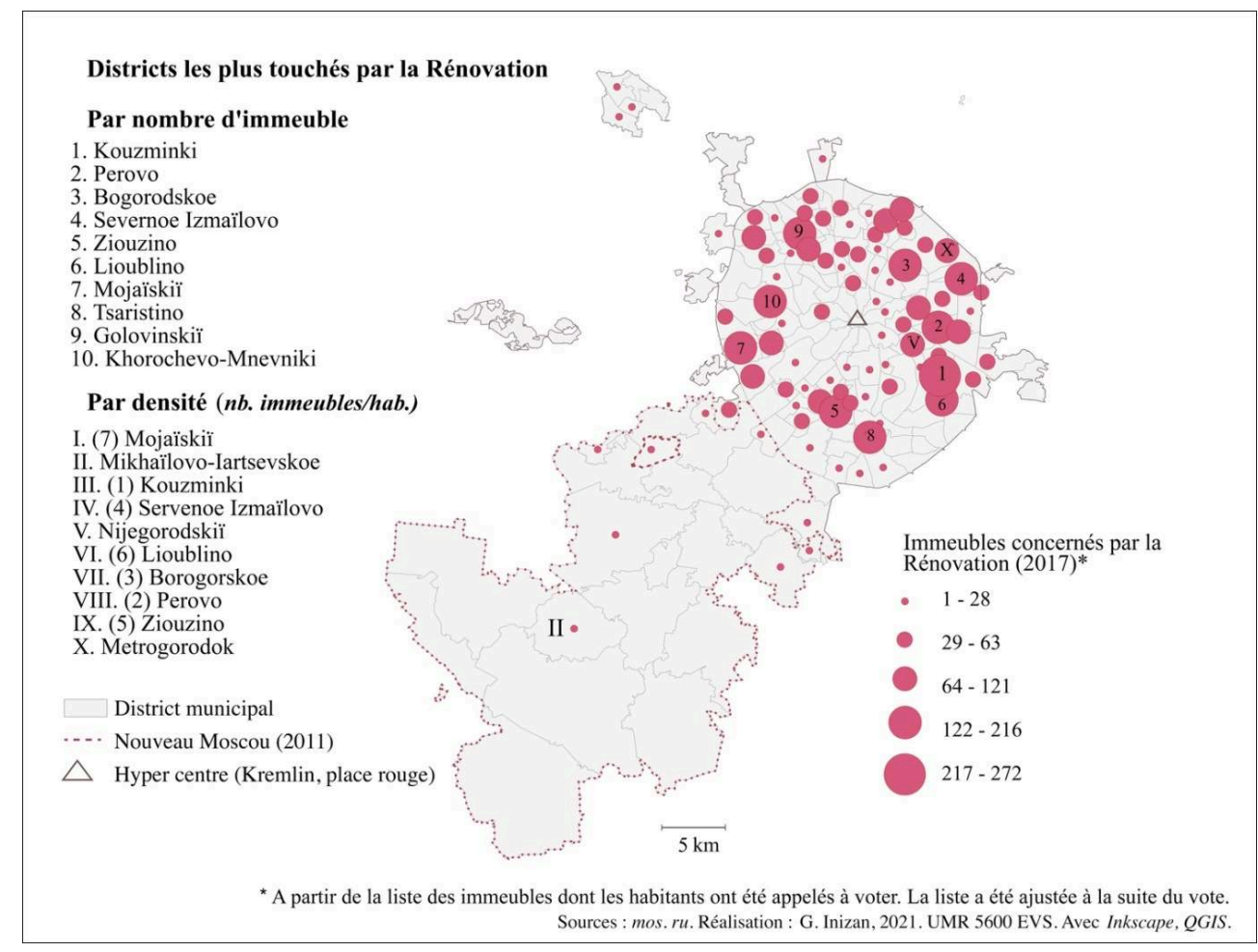

Auteur : G. Inizan, 2021.

\section{Des cibles nouvelles (et approximatives) de la mairie Sobianine}

9 La question de la dégradation des immeubles khrouchtchéviens n'est ni nouvelle, ni propre à la capitale russe. Elle résonne plus largement avec la question du devenir des grands ensembles construits dans les pays d'Europe centrale et orientale. Traversés par la transition postsocialiste, ils ont connu de profondes mutations, parfois été les cibles de programme de réhabilitation (Coudroy de Lille, 2004; Szafrańska, 2015) puis de démolition, à l'instar du StadtUmbau Ost en ex-Allemagne de l'Est (De Gaspérin, 2006 ; Florentin, 2011). Les immeubles khrouchtchéviens s'inscrivent dans cette trajectoire. A 
leur architecture sommaire s'est ajoutée la faiblesse de l'entretien des parties communes causée par le manque de moyens des structures vouées à leur maintenance à la fin de la période soviétique et après la privatisation. Dans les années 1990, les premières réponses politiques passaient par des programmes de réhabilitation ${ }^{7}$. Cependant, face à la dégradation avancée des immeubles (Krasheninnokov, 2003), des programmes de démolition ont été mis en place localement. A Moscou, un programme de démolition (Gunko et al., 2018) visant 1722 immeubles, initialement prévu entre 1999 et 2010, s'est finalement achevé il y a quelques mois seulement. D'autres villes russes ont été le cadre de programmes de démolition des immeubles khrouchtchéviens, comme la « rénovation » initiée à Saint-Pétersbourg en 2008 (Trumbull, 2014).

La conjoncture politique moscovite récente a conduit à un programme inédit par son ampleur. Arrivé après le limogeage de Iouri Loujkov (1992-2010), le maire Sergei Sobianine a d'abord privilégié d'autres dimensions du développement urbain: renouveau des espaces publics (Kalyukin, Borén et Byerley, 2015; Trubina, 2020), élargissement du réseau de transport et des frontières administratives de la ville avec le Nouveau Moscou (Argenbright, 2018). Dans ce contexte, la démolition des immeubles khrouchtchéviens apparaît comme une étape ultime pour « moderniser » la ville, selon l'expression régulièrement reprise dans les textes de la municipalité. Officiellement, l'urgence du programme est en effet justifiée par l'obsolescence imminente des immeubles. Dans le décret précisant le déroulement du programme, il est en effet expliqué que l'ambition est d'«éliminer le déséquilibre du développement urbain accumulé ces dernières décennies et empêcher l'apparition d'ici dix ou quinze ans d'un fonds de logements vétustes à Moscou» ${ }^{8}$. Elle est aussi un moyen de redonner un élan au secteur de la construction immobilière. Les promoteurs impliqués sont en effet autorisés à vendre une partie des nouveaux appartements sur le marché, alors que les terrains sont particulièrement attractifs. Ceux-ci sont également permis par des investissements massifs de la municipalité, dont le budget est nettement plus important que ceux des autres villes du pays ${ }^{9}$. Symbole de la domination moscovite, la réplication du programme Rénovation est envisagée dans d'autres villes du pays (Zupan, Smirnova et Zadorian, 2021).

11 D'après ce même décret, les immeubles concernés sont ceux qui ont été construits dans le cadre de "la première vague de construction individuelle de logements", entre « 1957 et 1968 ». La carte représentant les démolitions prévues en mai 2019 (illustration 2) indique en effet que les districts les plus touchés forment un anneau péricentral autour du centre-ville de Moscou, qui épouse grossièrement le front d'urbanisation des années 1960 (Gunko et al., 2018). La plupart des districts les plus touchés ont en effet été inclus dans les frontières de la ville à l'occasion de l'élargissement de 1960 (comme Kouzminki, Perovo) ou y étaient déjà intégrés mais ont été radicalement transformés à cette époque par le plan de construction de logements (comme Bogorodskoe, Golovinskii). Le fait que certains districts du Nouveau Moscou soient peu densément peuplés (comme Mikhaïlovo-Iartsevkoe) explique qu'ils figurent parmi les immeubles les plus touchés en fonction de la densité d'immeubles par habitant.

12 Malgré ce cadrage légal et les similitudes des immeubles khrouchtchéviens, les cibles effectives sont relativement diverses et approximatives. Les différentes "séries" (immeubles reproduits à l'identique) visées ont des propriétés architecturales sensiblement différentes. Par exemple, le décret mentionne les séries I-515, 
préfabriquées en béton, et les séries I-513, aux murs de brique (illustration 3). Par la mention " ou immeubles assimilés ", le texte laisse une marge d'interprétation qui se traduit par l'inclusion d'immeubles variés. De fait, certains sont des «projets individuels » (individualnye proekty) qui n'étaient pas produits en séries. En outre, de façon plus marginale, des immeubles de périodes antérieures (années 1920 ou période stalinienne) ont aussi été inclus. Au contraire, certains immeubles khrouchtchéviens répondant aux critères officiels n'ont pas été ciblés. Justifié par l'impossibilité de reloger les habitants dans ces districts, cette exclusion questionne d'ailleurs le motif de l'obsolescence générale mis en avant pour des immeubles de même facture dans d'autres districts.

Illustration 2 - Immeubles concernés par la Rénovation et date de construction des immeubles bâtis à Moscou dans la partie centrale de Moscou (hors Nouveau Moscou, Zelenograd et enclave est de Kountsevo)

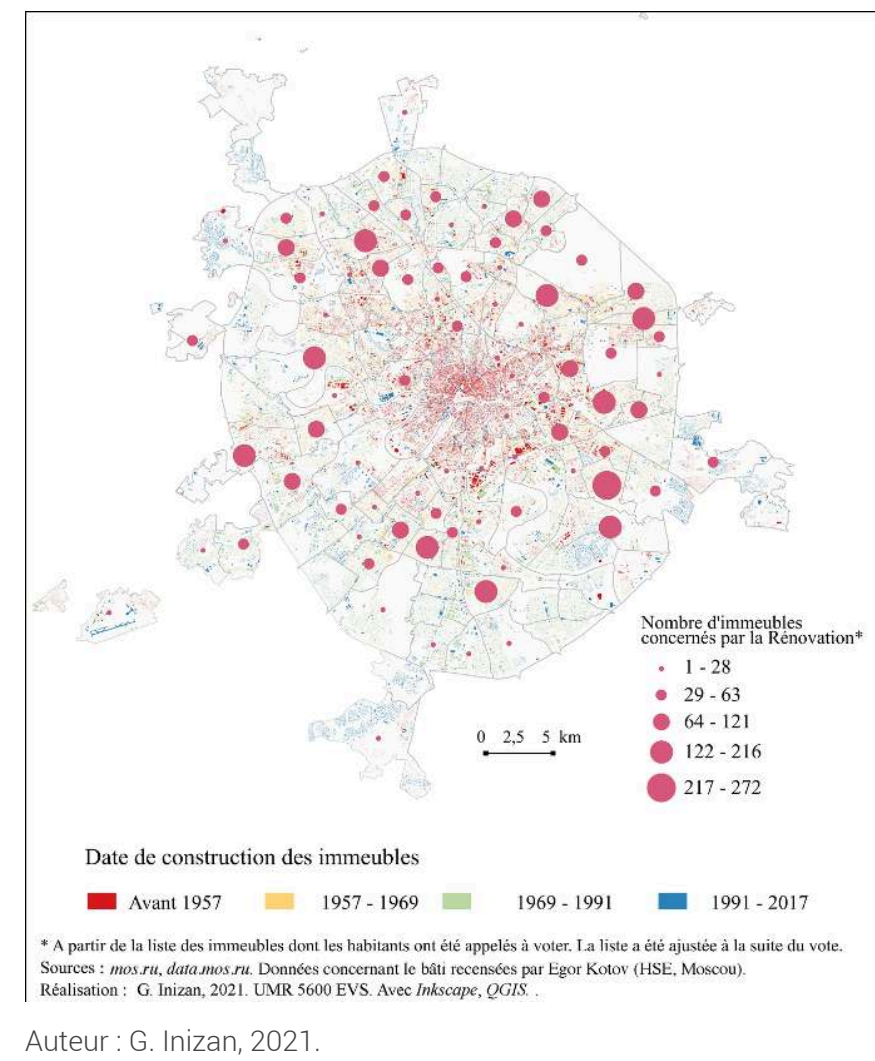


Illustration 3 - Immeubles d'habitation khrouchtchéviens

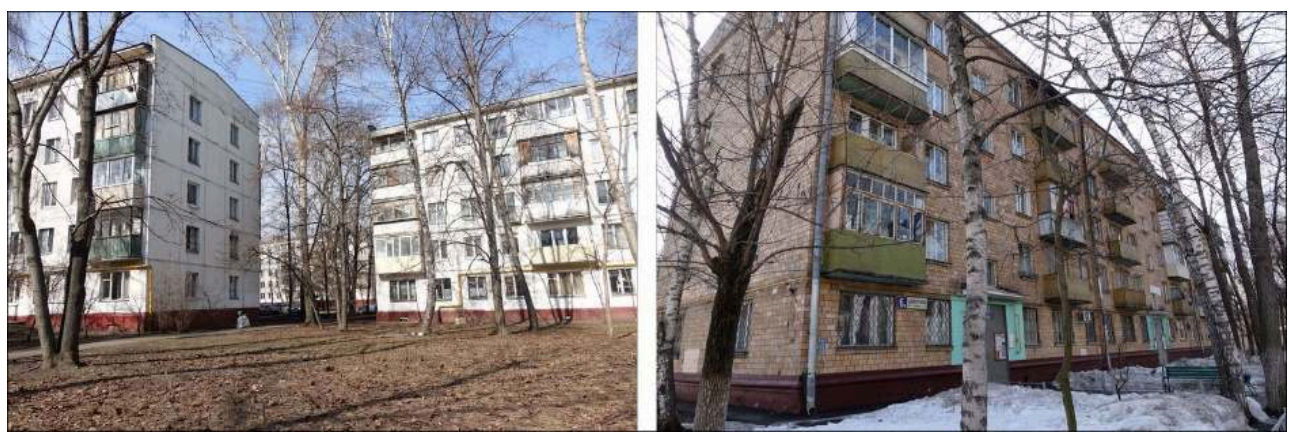

À gauche : en préfabriqué. À droite : en brique.

Auteur : G. Inizan, mai 2018 et avril 2019

\section{Des espaces habités de la transition}

13 La rénovation urbaine, comme politique de démolition-reconstruction impliquant le relogement des populations concernées offre un cadre particulièrement intéressant d'analyse du changement urbain. Comme politique, elle permet d'observer le fonctionnement de l'État ou des politiques urbaines (Epstein, 2013; Kirszbaum, 2018), des intérêts privés des promoteurs (Smith, 1979) ou des bailleurs (Bernt, 2019). Plus en aval, elle restructure l'organisation sociale de la ville, dont l'analyse conduit parfois à contredire les objectifs de mixité ou de déségrégation affichés par les instigateurs de la politique (Lelévrier, 2010; Bellanger et al., 2018). En tant qu'ensemble de négociations entre habitants et représentants des institutions, elle permet d'analyser les rapports de pouvoir qui se jouent au moment du relogement (François, 2014) ou lors de réunions locales entre habitants et représentants des institutions (Donzelot et Epstein, 2006 ; Lees et White, 2020).

Elle représente ainsi un événement intéressant pour comprendre la "transition", c'est-à-dire la mutation d'une ville socialiste à une ville postsocialiste, qui dans le cas moscovite est mue par une gestion néolibérale et autoritaire (Argenbright, 2016; Büdenbender et Zupan, 2017). Dans le domaine du logement, elle est notamment passée par l'instauration d'un marché et la privatisation des structures de maintenance et de la construction (Khmelnitskaya, 2019). Le stade précoce du programme Rénovation lancé en 2017 empêche de tirer des conclusions sur les conséquences sociales ou les transformations institutionnelles. En revanche, l'inachèvement de ce programme permet d'observer la transition en cours. La rénovation urbaine ouvre en effet une " phase d'inquiétude et d'espoir pour tous ceux qui sont touchés »; elle " fait lever un monde de représentations, d'attitudes envers le passé et l'avenir » (Coing, 1966). Bianca Botea la conçoit comme «un bouleversement des repères existants", de «la formulation de nouveaux modes d'intelligibilité » (Botea, 2014). C'est en m'intéressant à ces rapports aux immeubles khrouchtchéviens, tels qu'ils sont formulés, bouleversés et développés dans cette période d'entre-deux que je cherche à analyser la transition urbaine dans sa " rugosité » (Rolland et al., 2017).

Dans cette perspective, la notion d'appropriation de l'espace s'avère éclairante. Elle renvoie à un ensemble de pratiques et de représentations permettant l'usage ou le contrôle d'un lieu ou contribuant à produire un espace "à soi ", pour lequel est éprouvé un sentiment d'appartenance (Ripoll et Veschambre, 2005). La propriété 
juridique en est une dimension courante mais non systématique. Ainsi en Russie, la privatisation du logement a certes pu renforcer le sentiment d'appropriation (Attwood, 2012) mais plusieurs études ont aussi montré que ce n'était pas toujours le cas, en raison des forts droits d'usage associés à l'occupation d'un logement municipal (en partie hérités de la période soviétique) (Chaigneau, 2012), des coûts d'accès à la propriété ou des charges associées (Zavisca, 2012).

Les mobilisations sociales peuvent aussi peuvent contribuer à l'appropriation d'un espace, tout comme elles en dépendent (Ripoll, 2005). À Moscou, le fort contrôle policier de l'espace public rend particulièrement difficile, et par-là même symbolique, son appropriation à des fins contestataires (Poupin, 2012; Abramova, 2015). Les grandes manifestations dans les lieux centraux ne sont les seules façons de contester. L'appropriation des espaces résidentiels, par une familiarisation avec les structures juridiques qui le régissent, par des résistances localisées face à des accaparations illégales ou des projets imposés d'« en haut » (pouvoirs publics ou promoteurs), ou par la réalisation de projets culturels ou artistiques permet aussi de s'émanciper en partie de structures économiques, politiques et sociales inégales. Comme plusieurs travaux l'ont montré depuis une quinzaine d'années (Clément, Myriasova et Demidov, 2010; Carton de Grammont, 2013; Richard, 2014; Argenbright, 2016; Fröhlich, 2019), à Moscou, l'action collective, voire "politique", est aussi une affaire de résistances quotidiennes et locales, notamment dans le domaine du logement ou de l'aménagement urbain.

Dans cette étude, l'appropriation de l'espace est identifiée au prisme de l'affection ou la désaffection pour l'espace habité qu'elles sont formulées par les habitants ${ }^{10}$. Elle est aussi analysée en regard des pratiques d'investissement de l'espace résidentiel à différentes échelles (appartement, immeuble, quartier) telles qu'elles sont rapportées et observées. L'accent est mis sur les permanences et les changements qui ont touché ces modalités d'appropriation des immeubles et quartiers résidentiels khrouchtchéviens, suite à l'annonce de la Rénovation.

\section{Les réceptions différenciées de la Rénovation, révélatrices de situations résidentielles inégales}

\section{Le vote, indicateur imparfait de réceptions contrastées}

18 Un premier indicateur de la réception du programme est le vote qui a été organisé par la municipalité quelques semaines après l'annonce du programme. Il appelait les habitants à répondre à la question suivante : êtes-vous pour ou contre la démolition de votre immeuble? Une liste d'adresses a été publiée sur le site internet de la municipalité, des lettres envoyées aux habitants et des messages d'information affichés sur les portes d'entrée des immeubles. Selon des conditions spécifiées dans le décret municipal ${ }^{11}$ du 2 mai 2017, le scrutin s'est déroulé du 15 mai au 15 juin suivant (avant d'être prolongé de deux semaines). Il définissait trois modalités possibles du vote : en ligne (via le portail ou l'application Citoyens Actifs ${ }^{12}$ ), au bureau de services du district municipal (MFTs) ou par la constitution d'un vote en assemblée générale de propriétaires (obchtchee sobranie sobstvennikov).

$19 \mathrm{Au}$ moment du vote, les conditions de relogement étaient en partie définies. Une deuxième lecture du programme à la Douma municipale au printemps 2017 avait 
permis aux habitants concernés d'obtenir quelques "garanties ", comme le fait d'être relogé dans le même district municipal (illustration 1). Cependant, les nouveaux appartements n'étant pas encore construits et la loi cadrant la réalisation de la Rénovation encore non promulguée, les propositions de relogement relevaient du projet. Le vote requérait ainsi plutôt un avis quant à la démolition de l'appartement occupé, plutôt qu'un positionnement par rapport à une proposition concrète de relogement.

Plusieurs éléments limitent l'interprétation des réceptions du programme par le vote. Tout d'abord, les personnes autorisées à voter ne correspondent pas parfaitement aux habitants, au sens des personnes occupant ces logements. Le décret municipal définissant les conditions du vote précise que pour participer, il faut avoir plus de douze ans, être propriétaire ou « détenteur d'un bail social » ${ }^{13}$. Ainsi, des propriétaires non occupants peuvent voter, alors que l'avis des locataires du marché privé n'est pas pris en considération. Par ailleurs, plusieurs fraudes ont été relatées et l'abstention (justifiée par l'absence du quartier pendant le scrutin, l'indifférence, la méfiance) rend invisible l'avis des habitants concernés. Enfin, les modalités de comptabilisation du scrutin (illustration 4) lissent les votes exprimés. Si plus des deux tiers des appartements dont la majorité simple des occupants s'étant exprimée a voté "oui ", alors l'immeuble est inclus dans le programme. Au sein d'un appartement, voire d'une famille, des occupants peuvent avoir voté de façon différenciée.

Compte tenu de ces limites, à l'échelle de la ville, environ $88,9 \%$ des immeubles sont restés sur la liste définitive des démolitions, ce qui témoigne d'une approbation certaine du programme. La possibilité pour des habitants dont l'immeuble n'avait pas été inclus à la liste initiale de l'ajouter au programme par la décision d'une assemblée générale de propriétaires a par ailleurs entraîné une augmentation du nombre des démolitions prévues ${ }^{14}$. Dans le district étudié, Bogorodskoe, l'opposition atteint environ $30 \%, 51$ des 184 immeubles initialement inclus ayant été exclus. Elle est principalement concentrée dans les immeubles de brique (souvent mieux isolés, aux appartements plus confortables et mieux valorisés sur le marché de l'immobilier). Près de la moitié des immeubles en brique ont en effet été exclus suite au vote ( 43 sur 89 , soit $48 \%$ ), pour seulement $8 \%$ des immeubles préfabriqués en béton ( 8 sur 95 ), à l'architecture initialement plus modeste et encore plus exposés à la dégradation matérielle. 
Illustration 4 - Inclusion ou exclusion de l'immeuble dans le programme Rénovation selon le résultat du vote

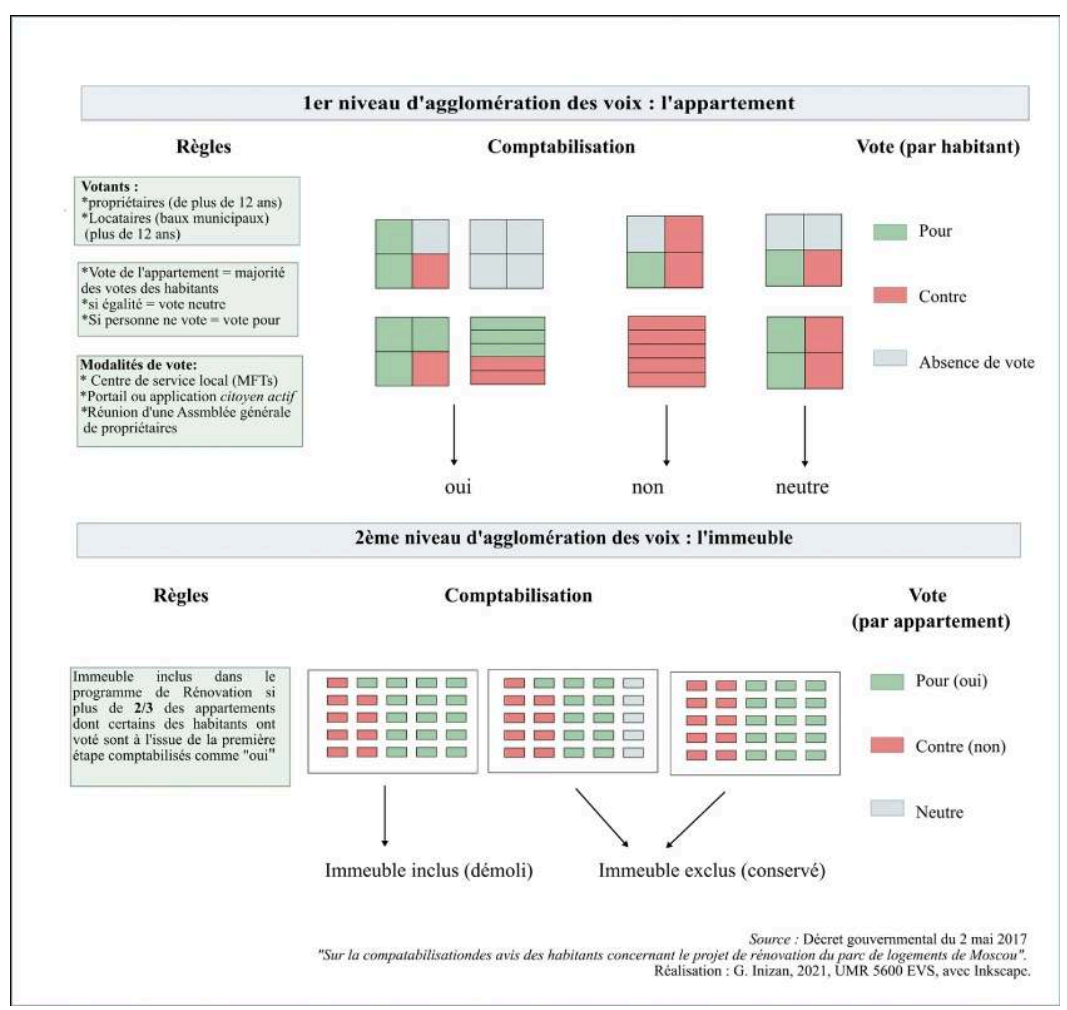

Auteur : G. Inizan, 2021

\section{Des projets de vie dans le projet de ville : situations résidentielles inégales face à la Rénovation}

Si les résultats du vote fournissent des indications sur les réceptions différenciées du programme, ils ne témoignent pas des motivations qui poussent à espérer ou à craindre la Rénovation. Or ces réactions s'inscrivent dans des situations et trajectoires différenciées. J'en propose ici une classification qui prend en compte le statut d'occupation du logement, le sentiment d'appropriation et la réaction à la Rénovation. Elle est construite comme une typologie des entretiens réalisés ${ }^{15}$, qui ne donc rend pas compte de l'ensemble des situations dans le district. Elle masque nécessairement la diversité des situations individuelles, qu'elle n'épuise pas. Elle laisse de côté en particulier les locataires occupant (qui n'ont pas pris part au vote) et les propriétaires non occupants. Par ailleurs, puisqu'elle vise à traduire des réactions différenciées à l'annonce du programme, elle ne traduit pas les changements de positionnements qui ont pu avoir lieu après. Néanmoins, elle donne à voir diverses perspectives sur la Rénovation liées aux inégalités résidentielles dans un même quartier. Quatre types de situations ${ }^{16}$, illustrées ci-dessous par de courts récits de trajectoires résidentielles, se dégagent en effet.

Situations résidentielles et réactions face à la Rénovation au travers de résumés de situations décrites pendant les entretiens, mars-juin-2019 ${ }^{17}$

Nikolaï (33 ans), propriétaire "par privatisation", occupe un appartement deux-pièces dans un immeuble khrouchtchévien préfabriqué en béton, avec sa femme et leur fille. Ils ont fait beaucoup de travaux mais ne se trouvent pas à leur 
aise dans cet appartement qui est sonore, mal isolé et au sein duquel ils ne peuvent pas faire les aménagements qu'ils souhaiteraient (du fait de la surface limitée et de l'architecture). Il ne veut pas déménager car il ne voit pas de meilleure alternative accessible sur le marché, mais ne se plait pas dans son appartement. La Rénovation est une occasion de quitter enfin ce logement pour quelque chose qui ne peut être que mieux, d'autant plus si cela lui permet de rester dans ce district qui lui plait.

Maria (34 ans), propriétaire "par achat ", vit dans un appartement une pièce d'un immeuble khrouchtchévien préfabriqué avec son mari et sa fille. Selon elle, cet achat était largement contraint par un budget serré. Elle est attachée au district mais les conditions de logement sont trop inconfortables et elle se réjouit donc de la Rénovation.

Alina (55 ans), propriétaire par « achat ", dirigeante d'un salon de coiffure, vit dans un immeuble khrouchtchévien en brique, au troisième étage, dans un appartement trois pièces qu'elle et son ex-mari ont acheté il y a dix ans, après avoir économisé plusieurs années. Ils l'ont complètement rénové. Elle cherchait à acheter dans ce type de quartiers et dans ce type d'immeubles. Elle est très attachée à son appartement dans lequel elle vit aujourd'hui avec sa fille. Le vote a finalement permis d'exclure l'immeuble du programme, mais elle a peur qu'une décision des autorités annule cette décision.

Evgenya (63 ans), propriétaire "par privatisation", retraitée, habite un appartement deux-pièces dans un immeuble khrouchtchévien préfabriqué. Elle a hérité de cet appartement de sa grand-mère, qui s'était vu attribuer ce logement après la démolition de sa petite maison en bois lors du programme khrouchtchévien, à Bogorodskoe. Elle y vit seule, mais y accueille régulièrement ses petits-enfants et est fortement opposée à la Rénovation.

Premièrement, certains habitants occupant un appartement qui leur - ou à leur famille - a été alloué pendant la période soviétique (soit par une entreprise étatique, soit par le soviet local) ont le sentiment de subir l'héritage de ce logement où ils se sentent assignés. Ils souhaiteraient vivre dans un lieu qui corresponde davantage à leurs besoins. En dépit_d'éventuels travaux, l'architecture de l'appartement (comme les murs porteurs) ou de l'immeuble (absence d'ascenseur) limitent la possibilité d'aménager l'espace à leur convenance. Ils n'ont pas la capacité ou l'envie de déménager en achetant un autre appartement. Considérant que le logement neuf sera forcément mieux, ils voient la Rénovation comme une opportunité.

Ce sentiment d'assignation est aussi éprouvé par des habitants ayant acheté leur appartement sur le marché de l'immobilier (depuis la privatisation du logement), mais pour qui cet achat était largement contraint, ce qui les conduit à subir des conditions de logement médiocres. Pour ces habitants, propriété privée et sentiment d'appropriation, au sens d'attachement affectif, sont déliés. La Rénovation surgit comme la possibilité d'une mobilité résidentielle ascendante et souhaitable.

Troisièmement, des propriétaires, qui ont acheté leur appartement sur le marché de l'immobilier, sont opposés à la Rénovation. Cet achat a représenté des efforts financiers importants, le fruit d'années d'économies. Souvent finalement permis par un élément facilitateur (héritage, contraction d'un emprunt immobilier...), il apparait comme un choix. L'appartement, où des aménagements intérieurs ont été faits, est présenté comme un espace "d'indépendance » et de "liberté ». Cette association entre liberté, propriété et appropriation a brutalement été mise à mal avec l'annonce du programme et de la démolition potentielle de l'immeuble, très négativement reçue car perçue comme une dépossession imposée.

26 Enfin, des habitants qui occupaient ou dont la famille occupait le logement pendant la période soviétique, y restent très attachés et sont donc opposés au programme. Ce 
logement, qui leur a été attribué selon les règles en vigueur à l'époque représente une valeur sentimentale, matérielle, et offre un cadre de vie que leur position sociale actuelle ne leur aurait pas permis d'acquérir en achetant un logement sur le marché. L'appropriation du logement, produite par le temps long de l'habiter, souvent associée à des souvenirs familiaux, joue un rôle majeur et est parfois renforcée par une privatisation (après 1992).

Sans prétendre à l'exhaustivité, cette classification permet néanmoins de montrer que la Rénovation a été perçue de façon différenciée en raison des parcours résidentiels inégaux des ménages concernés. Ainsi les immeubles khrouchtchéviens, imprégnés d'ambivalentes "cristallisations du passé » et de diverses «sédimentations des rapports sociaux » (Carton de Grammont, 2013) ${ }^{18}$ sont des espaces très inégalement appropriés, soudainement soumis au même « événement » (Coing, 1966).

\section{Quelles (ré)appropriations de l'espace habité ? (Im)mobilisations individuelles et collectives}

\section{Le programme Rénovation, inhibiteur et catalyseur d'appropriations de l'espace habité}

L'annonce de la Rénovation a façonné un " espace-temps » (Botea, 2014) où les modalités d'appropriation de l'espace habité (achat ou privatisation, investissements matériels, temps consacré à la vie de l'immeuble ou du quartier) ont été affectées. Certaines ont été fortement fragilisées par la Rénovation et l'incertitude de son calendrier. Alors que les premières listes d'immeubles ont été dévoilées quelques semaines après l'annonce des résultats, la majorité des habitants concernés ne sait pas encore, à l'heure où sont écrites ces lignes, quand le déménagement aura lieu. Ainsi, la motivation des démarches et pratiques visant à s'approprier un «chez soi » a été vivement questionnée : à quoi bon s'approprier son appartement ou son immeuble, s'ils sont condamnés à disparaître ? Son quartier, s'il doit être reconstruit? Et surtout, comment le faire si on ne connaît pas l'échéance de cette disparition? Cela se manifeste par exemple à travers les réflexions autour des travaux dans l'appartement, dont la rentabilité ou l'usage attendu sont brutalement interrogés. Au niveau de l'immeuble, le programme de réparations lourdes (kapremont) devient l'objet de questionnements : que va devenir l'argent investi? Faut-il espérer des réparations si l'immeuble est promis à la démolition? Par ailleurs, la gestion de ces réparations est parfois découragée par l'investissement nécessité par la Rénovation (extraits d'entretiens cidessous).

Travaux et réparations face à l'incertitude de la Rénovation

"Combien investir dans un appartement, dont on estime qu'il sera démoli dans un mois ? Mais si ce n'est pas dans un mois, mais dans dix ans... Alors ça vaudrait le coup de le faire » (entretien avec une habitante de Bogorodskoe, juin 2019).

«Le programme de réparations lourdes [kapremont] a été mal fait... Mais je n'ai même plus la force de ... Enfin, tu comprends. Après cette guerre de la Rénovation, j'ai tout juste commencé à me reposer» (e ntretien avec une habitante de Bogorodskoe, juin 2019).

À l'inverse, diverses mobilisations ont été déclenchées par l'annonce de la Rénovation. Certaines sont liées aux modalités institutionnelles du programme, comme le vote ou la participation aux « audiences publiques » invitant les habitants à se prononcer sur les 
aménagements prévus dans le district. D'autres émanent de la volonté d'échapper à l'incertitude: certains habitants ont ainsi rapidement vendu leur appartement ou cherché de le faire. L'annonce a aussi généré des actions motivées par la volonté de préserver l'immeuble de la démolition. Plusieurs opposants à la Rénovation décrivent leur appartement comme un abri contre les tensions extérieures, les incertitudes causées par les difficultés économiques, une description qui fait écho à la conception "moderne » de la maison, "un endroit dépourvu de la peur et de l'anxiété, un lieu supposé hors de portée des processus sociaux, politiques et naturels » (Kaika, 2004, p 266). L'annonce de la Rénovation a brusquement troublé cette quiétude. L'investissement dans la vie de l'immeuble ou du district, et en particulier l'engagement en "politique» (terme employé par les habitants), est alors présenté comme la réponse contrainte à cette intrusion brutale dans la sphère privée (citations ci-dessous). À cet égard les oppositions à la Rénovation s'inscrivent dans la lignée de nombreuses mobilisations en Russie, suscitées par des difficultés ou des injustices de la vie quotidienne face auxquelles des gens "ordinaires" deviennent "activistes " (Clément, Myriasova et Demidov, 2010).

\section{L'engagement "politique" contraint face à la Rénovation}

"Je ne voulais pas de la politique... Voilà, j'ai ma vie. Pourquoi on vient déranger ma vie privée? C'est mon droit, en tant que personne. J'ai ma petite famille, mes centres d'intérêt, mes amis. C'est ça qui m'intéresse. Pas de me battre avec vous. Mais bon. J'ai dû le faire » (entretien avec une habitante de Bogorodskoe, mai 2019). «Oui, beaucoup de gens disent: «la politique, je ne m'en occupe pas, ça ne m'intéresse pas », mais quand la politique débarque dans ta maison, tu commences à t'intéresser, à ce qui se passe, que tu le veuilles ou non, tu commences à t'y intéresser, tu dois comprendre, et faire en sorte d'apprendre, de maîtriser ce qui arrive » (entretien avec une habitante de Bogorodskoe, juin 2019).

\section{Des immeubles « ordinaires » au « village » contestataire?}

Ces mobilisations individuelles ont conduit à des (ré)appropriations collectives des immeubles et du quartier, en particulier autour de la mobilisation «contre » le programme, c'est-à-dire opposées à sa finalité (la démolition) ou à ses modalités de mise en œuvre. Les appréhensions et critiques portent sur les motivations du programme (enrichissement des promoteurs au détriment des habitants, opération politique de la municipalité), ses conséquences (densification et "ghettoïsation») ou ses modalités (opacité du vote, violation du droit de propriété). Ces inquiétudes, mues en accusations lors des mobilisations collectives, ont évolué au fil des annonces. Par exemple, si la crainte de la "déportation », exprimée au début de la mobilisation, a été en partie dissipée par l'annonce de la garantie du relogement dans le même district municipal, des témoignages mécontents concernant les premiers relogements ont soulevé des appréhensions quant à la qualité des nouveaux appartements.

31 Suite à l'annonce du programme, les habitants soucieux se sont rencontrés dans les cours, les halls d'entrée, les cages d'escaliers des immeubles. Des groupes ont été créés sur les réseaux sociaux ou les messageries instantanées pour partager des informations sur le programme ou organiser des actions pour le contester. Dans le district de Bogorodskoe, la mobilisation a été en partie portée par des propriétaires ayant acheté leur appartement récemment, mais implique aussi des gens qui ne sont pas directement concernés. Plusieurs d'entre eux, plus de deux ans après leur rencontre au printemps 2017, continuent de fréquenter les voisins connus pendant l'épisode 
"Rénovation ", surnommés " amis » de la Rénovation, vivant dans un même "village ". Les réseaux sociaux, les groupes de voisins servent désormais de supports à des échanges sur des sujets aussi divers que les réparations lourdes des immeubles, la gestion des parties communes, les problématiques de délimitation des terrains en copropriété, les activités associatives du quartier... Ainsi, si la vive contestation est souvent présentée comme un épisode achevé (après la publication de la deuxième liste, suivant le vote, à l'été 2017) et s'est essoufflée par la «fatigue » des habitants impliqués, elle a généré la création de réseaux de voisinage, socles de participation à la vie du quartier et des immeubles, qui perdurent.

La mobilisation est aussi passée par une importation de la Rénovation dans le champ politique. Les opposants ont pris part à des manifestations localement, mais aussi dans le centre-ville. Le rassemblement le plus important, le 14 mai 2017, aurait réuni environ 20000 personnes. Ils ont aussi investi la sphère politique locale. À Bogorodskoe, plusieurs députés d'opposition du conseil municipal, élus lors des élections de septembre 2017, s'étaient fait connaître localement lors des mobilisations liées à la Rénovation, un phénomène commun à d'autres districts moscovites (Zhelnina, 2019b). Si ces députés municipaux ont essentiellement une fonction représentative, sur les réseaux sociaux et lors de réunions plus ou moins formelles, ils continuent d'aider les habitants au sujet des problématiques de gestion des immeubles ou d'aménagement du quartier. Ils se font aussi le relai d'informations autour de la politique municipale. Les échanges autour de la Rénovation qui participent à produire des lectures et des discours critiques sur les politiques urbaines conduisent ainsi à une "montée en généralité " (Clément, 2012) caractéristique de nombreux mouvements sociaux en Russie. Si les processus répressifs et la difficulté d'identifier des responsables expliquent en partie que les mobilisations locales sont souvent liées à des «situations et des problèmes inscrits dans le monde du quotidien " (Clément, 2012), celles-ci peuvent conduire à émettre des critiques plus larges du système. En outre, cela témoigne d'un autre sens de l'appropriation de l'espace: ce n'est pas uniquement un enjeu (défendre son immeuble), mais aussi une ressource (Ripoll, 2005): pour beaucoup d'habitants il s'agissait, en défendant les immeubles khrouchtchéviens, de revendiquer leur « droit » " à choisir ", "à décider "; pour des activistes ou opposants politiques locaux, de lutter contre le pouvoir municipal en défendant des espaces dont elle convoite l'usage.

\section{Renforcement et limites de l'appropriation collective de l'espace habité}

33 À l'échelle individuelle, des habitants ont acquis des compétences techniques, juridiques, militantes permettant de s'approprier davantage l'espace habité. À l'échelle $\mathrm{du}$ district, le développement de réseaux de voisinage et l'investissement du champ politique local, ont permis le développement du débat autour des aménagements du quartier, notamment ceux qui s'annoncent avec la Rénovation. Enfin, l'obtention de garanties, apparues entre la première et la dernière lecture à la Douma de la loi Rénovation (août 2017), ou bien l'exclusion de certains immeubles du programme, sont perçues par certains habitants comme des victoires de la mobilisation.

Ces formes de réappropriation sont cependant limitées. Non seulement elles concernent une minorité d'habitants, mais aussi elles se heurtent en plus parfois aux réceptions positives du programme. Pendant le vote, certains habitants se sont 
organisés pour convaincre leurs voisins (par des tracts, des réunions, du porte-à-porte) de se prononcer en faveur ou contre le programme. Ces stratégies ont nécessité d'identifier les voisins au prisme du « pour » ou « contre », logique parfois traduite par l'établissement de listes matérialisant deux «camps». Du côté des «contre», les " pour » sont taxés de «fainéantise "; au contraire, les « pour » considèrent parfois les « contre » comme " pessimistes », ou " égoïstes ». Dans certains cas, les avis divergents sur la Rénovation se sont soldés par des échanges houleux, des formes d'évitement ou de conflits. La plupart des habitants disent " comprendre " leurs voisins qui n'ont pas le même avis eux, mais regrettent d'avoir à subir les conséquences de leur vote (entretiens ci-dessous).

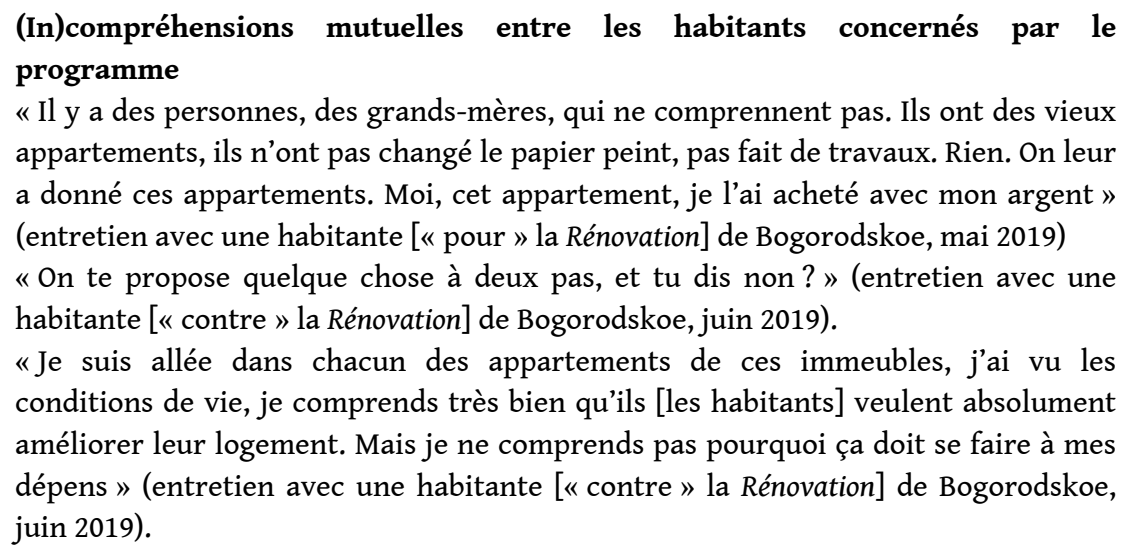

L'organisation institutionnelle du programme Rénovation, en particulier la mise en place d'un vote, témoigne donc d'une prise en compte de l'avis des habitants inédite à Moscou, mais aussi plus largement. Rares sont les projets de rénovation urbaine où les habitants sont appelés à voter. Cependant, en transférant l'apparente responsabilité du choix par une question imposée et sans alternative (entre la démolition et l'obligation de partir ou l'assignation à rester dans le même appartement), la municipalité a contribué à traduire des situations résidentielles inégales en intérêts convergents ou divergents face à la Rénovation.

\section{Conclusion}

Le programme Rénovation, lancé en 2017 à Moscou par le maire Sobianine, a placé les quartiers khrouchtchéviens, jusqu'alors terrains discrets des mutations postsoviétiques, au cœur des transformations de la capitale. La «transition » dont cette démolition participe est appréhendée ici à travers les réactions et résistances habitantes. Pour certains habitants, l'appartement occupé, fruit d'un achat contraint ou d'un héritage familial, matérialise l'impossibilité d'occuper un logement à la hauteur de ses besoins alors que pour d'autres, il est l'incarnation d'un «chez-soi » fortement approprié, la concrétisation d'un projet résidentiel ou d'un legs apprivoisé. Cet attachement d'une partie des habitants a contribué à nourrir une mobilisation qui a conduit à une réappropriation collective des immeubles et du quartier de façon pérenne. Cependant, l'annonce de la Rénovation a aussi produit des clivages. L'organisation institutionnelle du programme, notamment le vote, en transférant la responsabilité de la démolition aux habitants a traduit les réceptions variées de la Rénovation en intérêts contraires. 
écit de la Rénovation par les appropriations habitantes des immeubles et quartiers khrouchtchéviens contribue à l'étude de la transition post-soviétique à Moscou, telle qu'elle est vécue par ses habitants. Il laisse cependant des questions ouvertes, perspectives futures pour l'analyse de la Rénovation en cours, notamment autour de la conduite des relogements, des recompositions sociales et transformations institutionnelles induites, et de la conformité de l'expérience future du relogement aux craintes ou aux espoirs soulevés dans le temps de l'attente.

\section{BIBLIOGRAPHIE}

Abramova E., 2015. The Bolotnaya Square: Urban Design in Moscow Between Social Activities and Political Protests. Widok. Teorie i pratyki kultury wizualnej [En ligne]. URL: http:// widok.hmfactory.com/index.php/one/article/view/272/554 (consulté le 20 mai 2021).

Amestoy I., 2004. Les grands ensembles en Russie, de l'adoption d'un modèle à la désaffection. le cas de l'habitat khrouchtchévien. In Dufaux F., Fourcaut A., Le monde des grands ensembles. Paris, Creaphis, $251 \mathrm{p}$.

Argenbright R., 2016. Moscow under construction: city building, place-based protest, and civil society. Lanham, Lexington Books, 201 p.

Argenbright R., 2018. The evolution of New Moscow: from panacea to polycentricity. Eurasian Geography and Economics, vol. 9, n 3-4, p. 408-435.

Attwood L., 2012. Privatisation of Housing in Post-Soviet Russia: A New Understanding of Home? Europe-Asia Studies, vol. 64, n 5, p. 903-928.

Badyina A., Golubchikov O., 2005. Gentrification in central Moscow - a market process or a deliberate policy? Money, power and people in housing regeneration in Ostozhenka. Geografiska Annaler: Series B, Human Geography, vol. 87, n², p. 113-129.

Bellanger E., Collet A., Desage F., Gilbert P., 2018. Rénovation urbaine. L'espace comme remède à la question sociale ? Métropolitiques.eu [En ligne]. URL: http://www.metropolitiques.eu/ Renovation-urbaine-L-espace-comme-remede-a-la-question-sociale.html (consulté le 20 mai 2021).

Botea B., 2014. Expérience du changement et attachements : Réaménagement urbain dans un quartier lyonnais (la Duchère). Ethnologie française, vol. 44, n 3, p. 461-467.

Büdenbender M., Zupan D., 2017. The Evolution of Neoliberal Urbanism in Moscow, 1992-2015. The Evolution of Neoliberal Urbanism in Moscow. Antipode, vol. 49, n² 2, p. 294-313.

Carton de Grammont S, 2013. Savoir vivre avec son temps. Bref précis de cité-jardinage moscovite postsoviétique, comprenant quelques ruses symboliques de politique locale en période de libéralisation économique extrême, divers conseils et tours de mains sur l'art du bon voisinage avec les fantômes, ainsi qu'un menu requiem pour des efforts de bonheur. Thèse de doctorat en Anthropologie social et en Ethnologie (vol. 1). Paris, École des Hautes Études en Sciences Sociales, 827 p. 
Chaigneau A, 2012. La création des copropriétés en Russie. Un droit à l'épreuve de son usage. Revue d'études comparatives Est-Ouest, vol. 4, n 43, p. 35-69.

Churkina N., Zaverskiy S., 2017. Challenges of Strong Concentration in Urbanization: The Case of Moscow in Russia. Procedia Engineering, vol. 198, p. 398-410.

Clément K., 2012. Mobilisations citoyennes en Russie. Le quotidien au coeur des protestations. La vie des idées. [En ligne]. URL: https://laviedesidees.fr/Mobilisations-citoyennes-en-Russie.html (consulté le 20 mai 2021).

Clément K., Myriasova O., Demidov A., 2010. Ot obyvatelej k aktivistam : zaroždaûŝiesâ social'nye dviženiâ $v$ nynešnej Rossii [Des gens ordinaires aux activistes : les mouvements sociaux naissants dans la Russie contemporaine]. Moscou, Tri kvadrata, 690 p.

Coing H., 1966. Rénovation urbaine et changement social. L'ilot $n^{\circ} 4$ (Paris-13ème). Paris, Les Éditions Ouvrières, 296 p.

Coudroy de Lille L., 2004. Une idéologie du préfabriqué ? In Dufaux F., Fourcaut A., Le Monde des grands ensembles. Paris, Creaphis, p. 90-95.

De Gaspérin A., 2006, Rénovation et réhabilitation des grands ensembles dans les nouveaux Länder : quel avenir pour la ville socialiste? Revue Géographique de l'Est [En ligne], vol. 46, n 3-4. URL : http://journals.openedition.org/rge/1469

Donzelot J., Epstein R., 2006. Démocratie et participation : l'exemple de la rénovation urbaine. Esprit, juillet 2006, p. 5-34.

Epstein R., 2013. La rénovation urbaine : démolition-reconstruction de l'État. Paris, Presses de Sciences Po, 384 p.

Florentin D., 2011. Les Plattenbauten et le déclin : «Effet Plattenbau », politiques urbaines et représentations sociales dans les quartiers de grands ensembles à Leipzig. Géocarrefour, vol. 86, $\mathrm{n}^{\circ} 2$, p. 113-126.

François C., 2014. Au mépris des locataires: Infériorisation sociale et assignation résidentielle des relogés de la rénovation urbaine. Genèses, vol. 96, n 3, p. 86-109.

Fröhlich C., 2019. Urban citizenship under post-Soviet conditions: Grassroots struggles of residents in contemporary Moscow. Journal of Urban Affairs, vol. 42, $\mathrm{n}^{\circ} 2$, p. 188-202.

Golubchikov O., Makhrova A., 2012, Rossijskij gorod v usloviâh kapitalizma: social'naâ transformaciâ vnutrigorodskogo prostranstva [La ville russe sous le capitalisme : transformations sociales de l'espace intra urbain]. Vestnik Moskovskogo universiteta. Seriâ 5. Geografiâ, vol. 5, n 2, p. 26-31.

Gunko M., Bogacheva P., Medvedev A., Kashnitsky I., 2018, Path-Dependent Development of Mass Housing in Moscow. In Hess D.B., Tammaru T., van Ham M., Housing Estates in Europe. Cham, Springer International Publishing, p. 289-311.

Harris S., 2013. Communism on tomorrow street: mass housing and everyday life after Stalin. Washington, D.C, The Johns Hopkins University Press, 394 p.

Inizan G., Coudroy de Lille L., 2019, The last of the Soviets' Home: Urban demolition in Moscow, Geographia Polonica, vol. 92, n 1, p. 37-56.

Kaika M., 2004, Interrogating the Geographies of the Familiar: Domesticating Nature and Constructing the Autonomy of the Modern Home. International Journal of Urban and Regional Research, vol. 28, n², p. 265-286. 
Kalyukin A., Borén T., Byerley A., 2015, The second generation of post-socialist change: Gorky Park and public space in Moscow. Urban Geography, vol. 36, n 5, p. 674-695.

Khmelnitskaya M., 2015. The Policy-Making Process and Social Learning in Russia. The Case of Housing Policy. Londres, Palgrave Macmillan UK, 279 p.

Kirszbaum T., 2018. La rénovation urbaine aux Etats-Unis : une politique néo-libérale ? Metropolitiques.eu [En ligne]. URL: http://www.metropolitiques.eu/La-renovation-urbaine-auxEtats-Unis-une-politique-neoliberale.html

Kolossov V., O'Loughlin J., 2004. Comment Moscou se transforme en ville géante capitaliste. Revue internationale des sciences sociales, vol. 3, $\mathrm{n}^{\circ} 181, \mathrm{p} .463-477$.

Krasheninnokov A., 2003. The case of Moscow, Russia. In UN-Habitat, Global Report on Human Settlements. The Challenge of Slums. Londres, p. 195-228.

Lees L., White H., 2020. The social cleansing of London council estates: everyday experiences of accumulative dispossession. Housing Studies, vol. 35, $\mathrm{n}^{\circ}$ 10, p. 1701-1722.

Lelévrier C., 2010. La mixité dans la rénovation urbaine : dispersion ou re-concentration? Espaces et sociétés, vol. 140-141, $\mathrm{n}^{\circ} 1$, p. 59.

Makhrova A. G., Tatarintseva A. A., 2006. Razvitie processov džentrifikaciii rekonstrukciâ gorodskoj sredy centra Moskvy v postsovetskij period [Le Développement des processus de gentrification et de reconstruction de l'environnement urbain du centre de Moscou dans la période post-soviétique]. Regional"nye issledovaniâ, vol. 9, n 3, p. 28-42.

Makhrova A., Molodikova I., 2007. Land market, commercail real estate and the remolding of Moscow's urban fabric. In Stanilov K., The Post-socialist City. Springer, p. 101-114.

Marchand P., 2010. Atlas de Moscou. Paris, Éd. Autrement, 88 p.

Mosgorstat, 2018. Moskovskij statističeskij ežegodnik 2018. Èkonomika Moskvy v 2010-2017 gg [Annuaire statistique de Moscou, 2018. Economie de Moscou de 2010 à 2017]. Moscou, Mosgorstat, 188 p.

Olivier de Sardan J.-P., 2008. La rigueur du qualitatif : les contraintes empiriques de l'interprétation socio-anthropologique. Louvain-La-Neuve, Academia-Bruylant, 365 p.

Poupin P., 2012. "Démocratie dirigée" et manifestations protestataires à Moscou : quelle partition joue la police? The Journal of Power Institutions in Post-Soviet Societies [En ligne]. URL:: http:// journals.openedition.org/pipss/3921

Reid S. E., 2018. Palaces in our hearts: caring for Khrushchevki. In Großmann T, Nielsen P., Architecture, Democracy and Emotions: The Politics of Feeling Since 1945. Abingdon, Oxon, Routledge, p. 141-174.

Richard H., 2014. Comment les Russes sont-ils devenus (co)propriétaires? Thèse pour le doctorat de Sciences Politiques. Lyon, Institut d'Études Politiques de Lyon, 627 p.

Richard H., 2008. Du troc au marché : le marché immobilier à Moscou. Autrepart, vol. 48, nº 4, p. 87-99.

Richard H., 2013. Gérer son immeuble comme une « affaire » ? Dispositions économiques et réticences à la « mise en copropriété » de l'habitat collectif en Russie postcommuniste. Politix, vol. 101, $\mathrm{n}^{\circ} 1$, p. 161-182.

Ripoll F., 2005. S'approprier l'espace... ou contester son appropriation? Une vue des mouvements sociaux contemporains. Norois, vol. 195, n², p. 29-42. 
Ripoll F., Veschambre V., 2005. Introduction. L'appropriation de l'espace comme problématique. Norois, vol. 195, n 2, p. 7-15.

Rolland L., Volin A., Coudroy de Lille L., Rivière-Honegger A., 2017. Les géographes français et la transition: une étude du changement spatial temporel. In Grisoni A., Sierra R., Nachhhaltigen und Transition : Konzepte [Transition écologique et durabilité : concepts]. Francfort, Campus Verlag, p. 297-321.

Smith D., 1996. The socialist city. In Andrusz G., Harloe M., Szelenyi I., Cities after socialism: Urban and Regional Change and Conflict in Post-socialist Societies. Oxford, Wiley-Blackwell, p. 70-99.

Smith N., 1979. Toward a Theory of Gentrification A Back to the City Movement by Capital, not People. Journal of the American Planning Association, vol. 45, $\mathrm{n}^{\circ}$ 4, p. 538-548.

Solopova N., 2021. La préfabrication en URSS: concepts techniques et dispositifs architecturaux. Berlin, DOM Publishers, 191 p.

Szafrańska E., 2015. Transformations of large housing estates in Central and Eastern Europe after the collapse of communism. Geographia Polonica, vol. 88, n 4, p. 621-648.

Szelényi I., 1983. Urban inequalities under state socialism. Oxford-New York, Oxford University Press, $168 \mathrm{p}$.

Trouchtchenko O., 1992. Les bénéficiaires de la réhabilitation du centre historique de Moscou. Sociétés contemporaines, vol. 11, $\mathrm{n}^{\circ} 1, \mathrm{p} .227-238$.

Trumbull N. S., 2014. Restructuring socialist housing estates and its impact on residents' perceptions: "Renovatsiia" of khrushchevki in St. Petersburg, Russia. GeoJournal, vol. 79, n 4 , p. 495-511.

Vendina O., 2013. Social' nyj atlas. Moskva za predelami istoričeskogo centra: gorodskie rajony $i$ ih social'nye lica [Atlas social de Moscou. Moscou au-delà du centre. Les districts de la ville et leurs dimensions sociales]. Moscou, Moskovskij urbanističeskij forum, 28 p. [En ligne]. URL: https:// issuu.com/mosurbanforum/docs/

Vendina O., 1997. Transformation processes in Moscow and intra-urban stratification of population. GeoJournal, vol. $42, \mathrm{n}^{\circ}$ 4, p. 349-363.

Zavisca J. R., 2012. Housing the new Russia. Ithaca. Cornell University Press, 264 p.

Zhelnina A., 2020. Engaging neighbors. Housing strategies and political mobilization in Moscow's Renovation. Thèse de doctorat. City University of New York, 258 p.

Zhelnina A., 2019a. Getto v horošem smysle» protiv «betonnogo getto»:rajonnye diskursyi renovaciâ v Moskve [le ghetto sans le bon sens du terme. Contre le ghetto de béton. Discours de districts et Rénovation à Moscou]. Gorodskie issledovaniâ i praktiki, vol. 4, nº 2, p. 21-36.

Zhelnina A., 2019b. National and Urban Politics Converge in Moscow: Will Local Activism Prevail? Metropolitics [En ligne]. URL: https://metropolitics.org/National-and-Urban-Politics-Converge-inMoscow-Will-Local-Activism-Prevail.html

Zotova M., 2012. Emergence of Gated Communities in Russia: Causes and Consequences. L'Espace Politique [En ligne], $\mathrm{n}^{\circ}$ 17. URL: http://journals.openedition.org/espacepolitique/2385

Zupan D., Smirnova V., Zadorian A., 2021. Governing through stolichnaya praktika: Housing renovation from Moscow to the region. Geoforum, vol. 120, p. 155-164. 


\section{NOTES}

1. Expression reprise à Steven Harris à propos des habitants originaires des immeubles khrouchtchéviens (Harris, 2013), elle-même empruntée à l'historienne Sheila Fitzpatrik, une des pionnières de l'histoire sociale soviétique.

2. Le terme "quartier" est ici employé pour le terme russe microraïon, forme urbanistique typique d'implantation des immeubles d'habitation sous Khrouchtchev et qui désigne aujourd'hui un ensemble d'immeubles d'habitation. Les microraïony sont situés dans des districts municipaux (munitsipalnyï raïon, sg.). Dans l'article, je parle donc de « district » quand je me réfère à l'entité territoriale administrative, et du « quartier khrouchtchévien » quand il s'agit d'évoquer un ensemble d'immeubles d'habitation khrouchtchéviens.

3. En juillet 2017.

4. Entretiens réalisés dans le cadre d'une recherche doctorale ; 23 entretiens (dont 18 avec des habitants directement concernés par le programme) ont été réalisés dans le district Bogorodskoe entre mars et juin 2019. Les entretiens étaient principalement axés sur le récit de vie : arrivée dans le district, rapport au logement, au quartier, réaction à la Rénovation. Treize autres entretiens ont été réalisés avec des habitants et/ou opposants en dehors du district (moins mobilisés ici). Les observations concernent des réunions informelles entre ces habitants, ainsi que des manifestations.

5. À Moscou, cette structure résidentielle était liée à l'allocation soviétique des logements, principalement organisée par les soviets locaux, les entreprises étatiques et de façon minoritaire, à partir des années 1960, par les coopératives. Certains quartiers ou types d'immeubles étaient voués aux élites du Parti, à des professions particulières, et d'autres aux ouvriers, mais souvent une certaine hétérogénéité sociale s'observait à l'échelle des immeubles (Vendina, 1997). La capitale russe connaissait ainsi des formes et des échelles de ségrégation communes aux villes socialistes, bien différentes de celles créées par le marché foncier et immobilier des villes capitalistes (Szelényi, 1983 ; Smith, 1996).

6. En 2017 le taux de propriété privée des appartements à Moscou était de 81,6\%. Sans disposer de chiffres sur les immeubles concernés, il est probable qu'il soit légèrement inférieur pour les immeubles khrouchtchéviens, puisque parmi l'ensemble appartements "privatisables ", le taux était de 76, 3\% en 2017 (Mosstat, 2018).

7. Plus récemment, l'option « réhabilitation » est celle qui a été privilégiée par le programme de «réparations lourdes» (kapremont: kapitalnyj remont), programme défini dans le code du logement depuis 2012, et structuré à l'échelle régionale. Les propriétaires contribuent à une caisse commune régionale, ou bien peuvent créer leur propre caisse.

8. Postanovlenie pravitel'stva Moskvy N 497-PP ot 1 avgusta 2017 g. Programme renovacii žiliŝnogo fonda $v$ gorode Moskve [Décret du gouvernement de Moscou N 497 PP du 1et août 2017, Programme de rénovation sur le parc de logements dans la ville de Moscou].

9. À titre indicatif, en 2014, le budget de la ville de Moscou est quatre fois supérieur à celui de la seconde ville de Saint-Pétersbourg et représente $20 \%$ de l'ensemble des budgets municipaux de Russie (Churkina et Zaverskiy, 2017).

10. Dans les entretiens, les questions suivantes étant notamment posées : "Aimez-vous votre quartier, votre appartement? « «Pourquoi ?» «Avez-vous le sentiment de choisir votre lieu de résidence ?», «Êtes-vous satisfait.e du programme de Rénovation?»). Plus largement, une place importante était accordée au récit de vit et à la description du rapport au logement et au quartier.

11. Postanovlenie pravitel'stva Moskvy N 245 -PP ot 2 maâ 2017 g. Ob učete mneniâ nâseleniâ po proetku renovacii žiliŝnogo fonda $v$ gorode Moskve [Décret du gouvernement de Moscou N 
245 PP du 2 mai 2017, Programme de rénovation sur le parc de logements dans la ville de Moscou].

12. Portail et application mobile lancés en 2015 par le maire Sobianine.

13. C'est-à-dire le plus souvent des personnes qui occupaient le logement pendant la période soviétique et n'ont pas fait la démarche de privatisation.

14. Environ $21 \%$ des immeubles inclus dans la seconde liste était issue de ce processus (Inizan et Coudroy de Lille, 2019).

15. Lors de l'enquête, j'ai cherché à m'entretenir avec des habitants aux situations les plus différenciées possibles. Cependant, des conditions du terrain (entrée par un réseau d'opposants, plus grande visibilité des habitants mobilisés) ont fait que les entretiens ont eu lieu majoritairement avec des habitants opposés au programme, propriétaires ayant acheté sur le marché de l'immobilier (10) ou habitant depuis la période soviétique ayant privatisé (4). La certaine « accumulation » de profils de propriétaires par achat opposés à la Rénovation, bien que minoritaires à l'échelle du quartier, a permis, par effet de «saturation » (Olivier de Sardan, 2008) d'identifier ce groupe rapidement. Au contraire, les entretiens avec des habitants favorables ont été plus rares (4), mais leurs récits recoupaient ceux d'autres habitants, retranscrits sur les réseaux sociaux, la presse ou rapportés indirectement dans les entretiens.

16. Dans sa thèse soutenue récemment (2020), la sociologue Anna Zhelnina propose une autre typologie qui classifie les réactions à la Rénovation en cinq catégories : skeptical rejection (rejet systématique de ce que propose le gouvernement), hopeful consent (attente liée à la présence sur d'autres listes de programmes), learning to mistrust (citoyens mal informé au départ, peu à peu critiques notamment $\mathrm{du}$ fait des autres), seing an opportunity (ceux qui ne pendaient pas déménager mais y voient une opportunité), pragmatic conformism (ne pas s'opposer au programme de peur de représailles de la part des voisins ou des autorités) (Zhelnina, 2020, chapitre 5).

17. Les prénoms ont été changés et les adresses non localisées par souci de confidentialité.

18. À propos des travaux d'Henri Coing et de Colette Pétonnet sur la rénovation urbaine en France.

\section{RÉSUMÉS}

Cet article propose de lire les dynamiques spatiales de la Moscou post-soviétique à travers les réceptions et réactions habitantes au programme Rénovation. Annoncé en 2017, celui-ci prévoit la démolition de plus de 5000 immeubles d'habitation khrouchtchéviens dans la capitale russe d'ici 2032. À l'appui d'une enquête menée dans un district moscovite, on montre que ces immeubles abritent aujourd'hui une forte variété de situations résidentielles, du fait de l'héritage de la structure résidentielle soviétique et $\mathrm{du}$ développement du marché de l'immobilier. En conséquence, si la Rénovation a généré des mobilisations collectives entrainant des formes de réappropriation l'espace habité, celle-ci a aussi été limitée par les intérêts contraires des habitants.

The spatial dynamics of post-soviet Moscow are analyzed through its residents' perceptions and reactions to the Renovation Program. Launched in 2017, it plans the demolition of more than 5000 khrushchevian housing blocks in the Russian capital by 2032. Based on a field study carried out in a muscovite neighborhood, this paper shows the large variety of housing situations, due to the heritage of the soviet housing structure and the development of the housing market. 
Therefore, Renovation has brought about local mobilizations that lead to a collective appropriation of space, but it has been limited by the opposing interests of the dwellers.

\section{INDEX}

Keywords : Moscow, urban renewal, inhabitant, post-soviet city, khruschevian housing blocks Mots-clés : Moscou, rénovation urbaine, habitant, ville post-soviétique, immeuble d'habitation khrouchtchévien

\section{AUTEUR}

\section{GUENOLA INIZAN}

Guenola Inizan, guenola.inizan@univ-lyon2.fr, est doctorante Université Lumière Lyon 2, membre de l'UMR 5600 EVS. Elle a récemment publié :

- Inizan G., Coudroy de Lille L., 2019. The last of Soviets' home: Urban demolition in Moscow. Geographia Polonica [En ligne], vol. 92, n 1, p. 37-56. DOI: https://doi.org/10.7163/GPol.0135

- Inizan G., 2020. En attendant la Rénovation : résistances habitantes dans un district moscovite. URBIA. Les cahiers du développement durable, Hors Série n 7, p. 93-108. 